\title{
Prospective THz Gyrotrons for High-Field Magneto-Resonance Spectroscopy
}

\author{
V.L. Bratman $^{1,2}$, A.E. Fedotov ${ }^{2}$, Yu.K. Kalynov ${ }^{2}$, and V.N. Manuilov ${ }^{2,3}$ \\ ${ }^{1}$ Ariel University, Ariel, Israel, v_bratman@yahoo.com \\ ${ }^{2}$ Institute of Applied Physics of Russian Academy of Sciences, Nizhny Novgorod, Russia \\ ${ }^{3}$ Nizhny Novgorod State University, Nizhny Novgorod, Russia
}

\begin{abstract}
A high-harmonic Large Orbit Gyrotron and a low-voltage gyrotrino placed inside a spectrometer cryomagnet enable greatly simplify terahertz systems for magneto-resonance spectrometers. Large Orbit Gyrotrons provide a powerful third-harmonic generation at frequencies of $1 \mathrm{THz}$ and $0.394 \mathrm{THz}$ in pulsed and $\mathrm{CW}$ regimes, respectively, at significantly lower magnetic fields than conventional gyrotrons. According to simulations the gyrotrino with the voltage of $1.5 \mathrm{kV}$ and frequency of $0.264 \mathrm{THz}$ can generate a power of tens of watts; a possibility to operate at such a low voltage is demonstrated in the existing gyrotron with three-electrode magnetron-injection gun.
\end{abstract}

\section{Introduction}

$\mathrm{THz}$ medium power gyrotrons are in high demand for high-field DNP-NMR spectroscopy. However, only few installations are now equipped with such fairly expensive generators. Large Orbit Gyrotrons (LOGs) and gyrotrinos may make this method accessible for many laboratories due to the considerable simplification of the gyrotron magnetic system.

Conventional gyrotrons commonly operate at the fundamental or second cyclotron harmonics. The latter allows using simpler cryomagnets with a halfvalue of the magnetic field. A greater effect can be achieved in LOGs, capable of selective operation even at the third and fourth harmonics due to applying an axis-encircling electron beam [1-5], in contrast to a hollow poly-axis beam in a conventional gyrotron.

Another option is integration of a compact gyrotrino in single cryomagnet with an NMR spectrometer [6, 7]. This is based on the proximity of the electron cyclotron frequency and the frequency of the paramagnetic resonance in a sample. The integration eliminates the need for an additional cryomagnet and a long $\mathrm{THz}$ transmission line with high losses. An exact frequency matching can be achieved at a very low gyrotron voltage, namely $1-2 \mathrm{kV}$ [6], or at special profiling the cryomagnet field [7].

\section{High-Harmonic Large Orbit Gyrotrons}

In a LOG cavity (Fig. 1), an axis-encircling electron beam can excite only co-rotating modes with azimuthal indices equal to the number of the resonant cyclotron harmonic [1-5]. This strong selection rule prevents the excitation of most of parasitic modes and makes it possible to operate at higher harmonics.
At the Institute of Applied Physics, LOGs, are being studied in parallel with conventional gyrotrons, for more than 20 years to obtain higher frequencies at lower magnetic fields. First experiments were carried out at high-current electron accelerators with moderately relativistic electron energy of $(250-400) \mathrm{keV}$. These LOGs selectively generated high output powers at harmonics $s=2-5$ and frequencies from $20 \mathrm{GHz}$ up to $0.4 \mathrm{THz}$ [5]. At relativistic energy, electron-wave coupling at high harmonics can be stronger than at the fundamental resonance, but a problem of neighboring harmonics discrimination is fairly complex. In addition, use of relativistic energy decreases the cyclotron electron frequency and reduces the frequency gain.

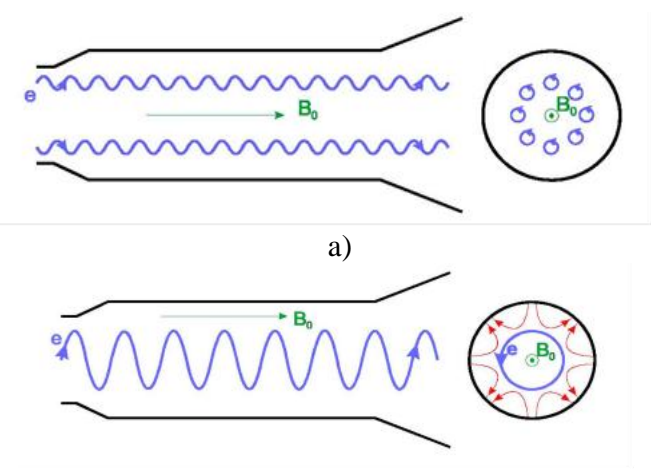

b)

Fig. 1. Electron beams in conventional gyrotron (a) and high-harmonic Large Orbit Gyrotron (b)

The further progress in development of LOGs was associated with reducing the operation voltage that enables replacing the accelerators by simpler high-voltage modulators, as well as with increasing the radiation frequencies. In the $80-\mathrm{kV}$ LOG (Fig. 2a) [4], a stable single-mode second- and third-harmonic generation with a power of $0.3-1.8 \mathrm{~kW}$ was obtained in $10-\mu \mathrm{s}$ pulses at four frequencies in the range of $0.55-1.00 \mathrm{THz}$ at magnetic fields $10.5-13.6 \mathrm{~T}$. A relatively low efficiency of this generator $\sim 1 \%$ was caused by great Ohmic losses in a long gyrotron cavity at $\mathrm{THz}$ frequencies. This LOG was used for scientific applications and for testing novel versions of $\mathrm{THz}$ cavities [5] aimed to enhance efficiency by decreasing Ohmic losses, as well as to make possible the fourth-harmonic operation.

An important step in LOG progress is a development of a $30 \mathrm{kV} / 0.7$ A gyrotron [5] with a $5 \mathrm{~T}$ cryomagnet designed for a CW operation (Fig. 2b). In this oscillator, an electron beam with a large pitchfactor of 1.5 is formed in a cusp gun and then the transverse electron velocity increases in an increasing 
magnetic field. The main scope of this setup is operation at the second, third and fourth harmonics at the

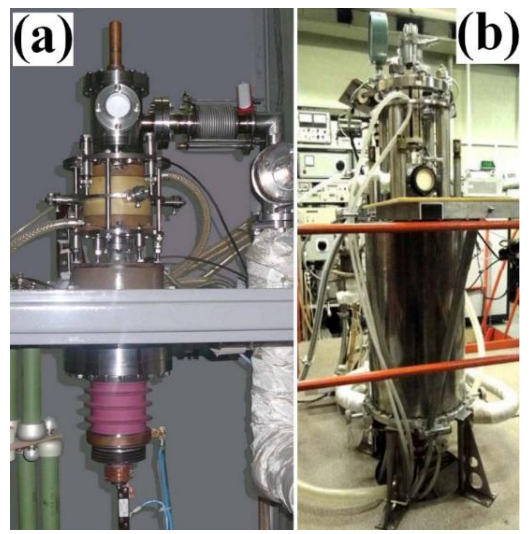

Fig. 2. LOGs with parameters $1 \mathrm{THz} / 80 \mathrm{kV}$ (a) and 0.394 $\mathrm{THz} / 30 \mathrm{kV}(\mathrm{b})$.

typical DNP frequencies of $0.26 \mathrm{THz}, 0.39 \mathrm{THz}$, and $0.52 \mathrm{THz}$ with the power level of hundreds of watts.

In a good accordance with simulations, experimental zones of excitation of the second-harmonic $\mathrm{TE}_{2,5}$ and third-harmonic $\mathrm{TE}_{3,7}$ modes are well separated by magnetic field. Generation with parameters $800 \mathrm{~W} / 0.267 \mathrm{THz}$ and $300 \mathrm{~W} / 0.394 \mathrm{THz}$ is obtained at the fields $B=5.02 \mathrm{~T}$ and $B=4.93 \mathrm{~T}$, respectively. Competition of these two modes is observed at intermediate magnetic fields. An increase in operating magnetic field up to $6.3 \mathrm{~T}$ and in the electron voltage up to $45 \mathrm{kV}$ should allow achieving frequencies up to $0.65 \mathrm{THz}$ at the fourth cyclotron harmonic [5].

\section{Gyrotrino}

Integration of a $\mathrm{THz}$ generator and an NMR spectrometer in single cryomagnet requires the exact matching of the DNP and gyrotron frequencies. Because of relativistic dependence of the cyclotron frequency on electron energy this is possible at an extremely low voltage of $1-2 \mathrm{kV}$ only [6]. The very possibility of operating at a such low voltage was verified in an existing CW gyrotron [8]. A three-electrode magnetron injection gun of the gyrotron was initially designed for a higher voltage; to obtain an acceptable electron pitch factor for the low voltage, a high positive potential was applied to a modulating anode while keeping a negative cathode potential in order to increase the transverse particle velocity in the emitter region and decrease the longitudinal velocity in a region between the anode and the cavity. As a result, a stable generation with the frequency of $0.25 \mathrm{THz}$ was observed at whole range of electron energies in the gyrotron cavity from $15 \mathrm{keV}$ down to $1.5 \mathrm{keV}$.

According to simulations [10], a gyrotrino with an operating $\mathrm{TE}_{6.2}$ mode, a voltage of $1.5-1.8 \mathrm{kV}$, a current of $200 \mathrm{~mA}$, a pitch factor of 1.2 and a small cavity length can provide a power up to $15 \mathrm{~W}$ at the frequency of $0.264 \mathrm{THz}$ with a required magnetic field about of $9.42 \mathrm{~T}$ (Fig. 3b). Since the gyrotron cavity is very close to the sample, a short transmission line is needed, that reduces the radiation losses and decreases a required $\mathrm{THz}$ power. A magnetic field disturbance induced by the low-voltage electron beam at the sam-
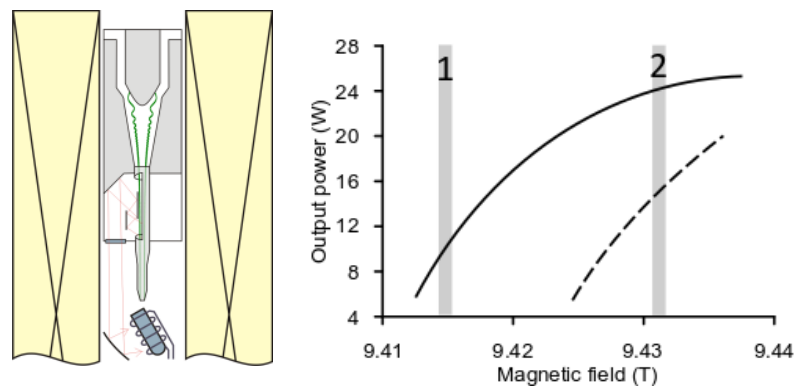

Fig. 3. a) Gyrotrino in cryomagnet of NMR spectrometer; b) calculated power vs. magnetic field for $1.5 \mathrm{kV} / 200 \mathrm{~mA}$ (solid line) and $2 \mathrm{kV} / 150 \mathrm{~mA}$ (dotted). Lines 1 and 2 show two regimes of gyrotron-DNP frequency matching.

ple is about of $10^{-8}$ and can be neglected. To save a limited space in the uniform magnetic field region, the radiation output from the gyrotron cavity can be directed toward the electron gun (Fig. 3a). Due to lack of space, the electron beam is to be collected in a cavity cut-off narrowing in a strong field [10].

The gyrotrino project was supported by Russian Science Foundation under grant No. 16-12-10445. Authors are grateful to M.Yu. Glyavin, A.P. Fokin, I.V. Osharin, and N.A. Zavolsky for collaboration.

\section{References}

1. Jory, $H$. Investigation of electronic interaction with optical resonators for microwave generation and amplification // Varian Associates, Palo Alto, CA, USA, R\&D Tech. Rep. ECOM-01873-F. 1968.

2. McDermott, D.B., Luhmann, Jr, N.C., Kupiszewski, A., and Jory, H.R. Small-signal theory of a large-orbit electron-cyclotron harmonic maser // Phys. Fluids. 1983. V. 26. No. 7. P. 1936-1941.

3. Lawson, W., Destler, W.W., and Striffler, C.D. Highpower microwave generation from a large-orbit gyrotron in vane and hole-and-slot conducting wall geometries // IEEE Trans. Plasma Sci. 1985. V. 13. No. 6. P. 444-453.

4. Bratman, V.L., Kalynov, Yu.K., and Manuilov, V.N. Large-orbit gyrotron operation in the terahertz frequency range // Phys. Rev. Lett. 2009. V. 102. No. 24. P. 245101.

5. Bandurkin, I.V., Bratman, V.L., Kalynov, et al. Terahertz Large-Orbit High-Harmonic Gyrotrons at IAP RAS: Recent Experiments and New Designs // IEEE Trans. Electron Devices. 2018. V. 65. No. 6. P. 2287-2293.

6. Bratman, V.L., Fedotov, A.E., Kalynov, Yu.K., and Samoson, A. THz Gyrotron and BWO Designed for Operation in DNP-NMR Spectrometer Magnet // J. Infrared, Millimeter, and THz Waves. 2013. V. 34. P. 837.

7. Sirigiri, J.R., Maly, T. Integrated high-frequency generator system utilizing the magnetic field of the target application // U.S. Patent No. 8,786,284, 22. Jul. 2014.

8. Glyavin, M.Yu., Denisov, G.G., Zapevalov, et al. High power terahertz sources for spectroscopy and material diagnostics // Phys.-Usp. 2016. V. 59. P. 595.

9. Bratman, V.L., Fedotov, A.E., Fokin, A.P., et al. Operation of a sub-terahertz $\mathrm{CW}$ gyrotron with an extremely low voltage // Physics of Plasmas. 2017. V. 24. P. 113105.

10. Bratman, V.L., Fedotov, A.E., Kalynov, et al. Numerical study of a low-voltage gyrotron ("gyrotrino") for DNP/NMR spectroscopy. // IEEE Transactions on Plasma Science. 2017. V. 45. No. 4. P. 644. 